\title{
Study on the Driving Force of Regional Land Use Change Based on GWR Model
}

\author{
Cai Dong ${ }^{1,2, a, *}$, Lin Man ${ }^{1, b}$ and Zhang Wei-lu, ${ }^{2, c}$ \\ ${ }^{1}$ Public Security Management Department, Jiangsu Police Institute, Nanjing, China \\ ${ }^{2}$ Key Laboratory of Police Geographic Information Technology, Ministry of Public Security, \\ Changzhou, China \\ ajickie_cd@126.com, ${ }^{\mathrm{b}} 1445346590 @ q q . c o m,{ }^{\mathrm{c}} \mathrm{m} 13082510121 @ 163 . c o m$ \\ *corresponding author
}

Keywords: Land Use Change, Driving Force, Geographical Weighted Regression.

\begin{abstract}
The study of land use or cover change is a comprehensive analysis of the quantity, quality and spatial distribution of land use. It is one of the core topics of the current global change research. With the support of spatial statistics theory, we studied Menghe Town Changzhou City, Jiangsu Province. With GIS as a means, we considered driving factors of land use spatial pattern of differentiation in the regression model simulation of land use patterns. We established and implemented based on GWR's simulation model of land use pattern. Then, we carry on the study on the driving force of regional land use change. It accurately reflected corresponding 1- and cover types of the true distribution based on GWR method of logistic regression model .We also consider the spatial instability of driving factors, it is able to reflect the spatial variation characteristics of various driving factors on land use types.
\end{abstract}

\section{Introduction}

Land use/Cover Change (LUCC) study is a comprehensive analysis on the changes of land use of the quantity and spatial distribution, has become one of the core themes of global change research. Driving force and mechanism of land change use is the analysis of land use changes' reasons and internal mechanism, which is the core issue of study on the driving force of regional land use change. It is made as the foundation of establishing land use dynamic model and land use temporal and spatial change prediction. By studying the driving force mechanism of land use change, it is found that the regularity of land use change, so we established land use change model to simulate land use change, which is the main line of LUCC research. At present, CLUE and improved CLUE$\mathrm{S}$ model widely used in home and abroad are based on the land use change's driving factors Logistic regression model and achieve the dynamic simulation of land use pattern.

With regard to analysis methods of driving factors, we gradually developed from the comprehensive discriminant model based on experience to quantitative analysis method based on mathematical statistics. The latter is the multivariate statistical analysis method. It analyses every external factors of land use changes in the contribution rate, so as to find out the external causes of land use change. The research shows that the method based on mathematical statistics can help to 
separate the main driving factors from the complex land use system, and determine the quantitative relationship between land use change and driving factors. Such as: Gobin (2002)used regression model to analyse the driving force of agriculture land use change and got the ideal result. Bai Wan qi et al(2004)used regression model to determine the quantitative relationship between land use change and driving forces. Bakkera (2005)using regression model found that the problem of land abandonment of Greek Lesvos island was caused by soil erosion. Zhang an Ding(2007)used transfer matrix ,correlation analysis and principal component analysis to study the land use change and its driving force in Longkou City. Xie Hua-lin et al (2008) analysed driving forces of land use change in farming pastoral zone, and the Logistic regression model of different land use types was established.

But now, when researching driving force of land use change based on mathematical statistics regression analysis and correlation analysis method, we carried on Global parameter estimation and didn't the spatial instability of land use change's effects. That is to say the influence of driving force factor and the spatial differentiation of the function, and the fact that the influence of a driving factor in different spatial location on land use pattern is different. GWR model is an extension of traditional regression framework and allows local but not global parameter estimation .Through additional expression changing parameters of correlation in itself and the heterogeneity of the object space, It reflects the regression samples of regression equation with the spatial differentiation and is able to follow the geographical space "hot spots". It is applied more and more wildly in the geographical elements of the spatial analysis and modelling. Thus, It was regarded as one of the most potential spatial statistical models. But its application research is still in the initial stage, especially the GWR model being applied to study the driving forces of land use change, and it still hasn't been seen in the literature at home and abroad at present.

\section{Overview of the Study Area}

Menghe Town, new North District, Changzhou City, located in the northwest, was established merging river with Meng river in October 2003. Menghe Town covers an area of 88.26 square kilometers, located in Changzhou West Ring Road and North Ring Road Interchange. With 238 and 338provincial highway cross over the border, Yangtze river to the east, south near the beijingshanghai railway, shanghai-nanjing expressway and 312 national road, north-west border with danyang, It is a gathering place which has a long history. Menghe Town, with convenient transportation, land use type is complete, terrain to the plains and hilly, dense water network, belongs to the North subtropical marine climate, year-round mild climate, abundant rainfall, four distinct seasons. Its topography, land use and other characteristics in Jiangsu province (especially in the central region of the Yangtze River Delta) has a certain representative of the township.

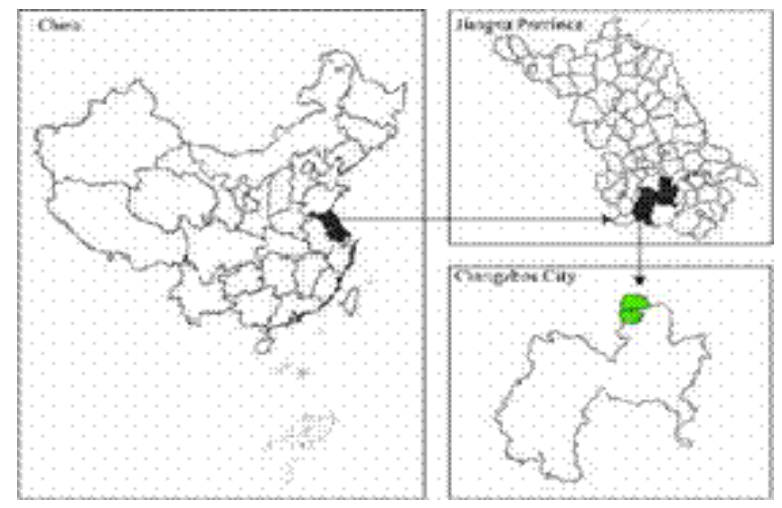

Figure 1: Location of Changzhou New District Menghe Town 


\section{Data Preparation}

Data used in the study of land is derived from land use maps of Menghe Town, New District, Changzhou City in 2005. We referred to TM remote sensing image's grid and reclassification of land use's current map in 2005 and cultivated land, forest land, construction land, ponds and unused five types of land cover types, the grid size being $100 \mathrm{~m} * 100 \mathrm{~m}$. At the same time, the use of Changzhou Xinbei District Menghe Town 25m digital elevation model, river system, soil, railway, the main highway, road, Hong station, the 2000 census data, selected in a relatively short time scale of a relatively stable natural geographical factors (elevation, slope degree, slope direction, social economic factors from the waters of the recent distance, the content of soil organic matter) and road traffic and population as a regional land use spatial pattern formation and evolution of the main driving factors.

\section{Land Use Change Driving Force Model Based on GWR Logistic}

GWR extends the traditional regression model $y_{i}=\beta_{0}+\sum_{k} \beta_{k} x_{i k}+\varepsilon_{i}$, so that parameter can do local estimation. Its model structure: $y_{i}=\beta_{0}\left(u_{i}, v_{i}\right)+\sum_{k} \beta_{k}\left(u_{i}, v_{i}\right) x_{i k}+\varepsilon_{i},\left(u_{i}, v_{i}\right)$ is the coordinate sample of I (as geographically weighted), $\beta_{k}\left(u_{i}, v_{i}\right)$ is the value of independent variables ${ }^{x_{k}}$ Coefficient continuous function $\beta_{k}(u, v)$ at the point I. According to the first law of geography, data close to the position of the observation have more influence on estimates of $\beta_{k}\left(u_{i}, v_{i}\right)$ than those far away from. Therefore, GWR in a weight observations focusing on the return process is no longer maintained, but with the proximity of the position $i$ is weighted, that is $\hat{\beta}\left(u_{i}, v_{i}\right)=\left(X^{T} W\left(u_{i}, v_{i}\right) X\right)^{-1} X^{T} W\left(u_{i}, v_{i}\right) Y$ among them:

$$
\begin{gathered}
X=\left[\begin{array}{cccc}
1 & x_{11} & \cdots & x_{1, k} \\
1 & x_{21} & \cdots & x_{2, k} \\
\cdots & \cdots & \cdots & \cdots \\
1 & x_{n 1} & \cdots & x_{n, k}
\end{array}\right] \quad W\left(u_{i}, v_{i}\right)=W(i)=\left[\begin{array}{cccc}
w_{i 1} & 0 & \cdots & 0 \\
0 & w_{i 2} & \cdots & 0 \\
\cdots & \cdots & \cdots & \cdots \\
0 & 0 & \cdots & w_{i n}
\end{array}\right] \\
\beta=\left[\begin{array}{cccc}
\beta_{0}\left(u_{1}, v_{1}\right) & \beta_{1}\left(u_{1}, v_{1}\right) & \cdots & \beta_{k}\left(u_{1}, v_{1}\right) \\
\beta_{0}\left(u_{2}, v_{2}\right) & \beta_{1}\left(u_{2}, v_{2}\right) & \cdots & \beta_{k}\left(u_{2}, v_{2}\right) \\
\cdots & \cdots & \cdots & \cdots \\
\beta_{0}\left(u_{n}, v_{n}\right) & \beta_{1}\left(u_{n}, v_{n}\right) & \cdots & \beta_{k}\left(u_{n}, v_{n}\right)
\end{array}\right] \quad Y=\left[\begin{array}{c}
y_{1} \\
y_{2} \\
\cdots \\
y_{n}
\end{array}\right]
\end{gathered}
$$

In the selection of the weighting function, this study used Gaussian function, that is, $W\left(u_{i}, v_{i}\right)=e^{-\frac{1}{2}\left(\frac{d_{i j}}{b}\right)^{2}}$ In the optimal bandwidth selection, this study used the minimum AIC method (Akaike Information Criterion, called AIC), which is calculated as: $A I C_{c}=2 n \ln (\hat{\sigma})+n \ln (2 \pi)+n \frac{n+\operatorname{tr}(s)}{n-2-\operatorname{tr}(s)}$

The subscript $\mathrm{c}$ indicates revised estimate, $\mathrm{n}$ is the sample size, the error term is estimated standard deviation, $\operatorname{tr}(\mathrm{s})$ is a trace of GWR's matrix $\mathrm{S}$, which is a function of the bandwidth. 
For comparison purposes and to simplify the calculation process, this study selected the last variable to enter the equation as the geography type's GWR driving force factor in OLS Logistic. We used GDAL library and Visual Studio.NET 2005 C \# language to implement GWR calculation process, achieving the regression results (Due to space constraints, we only show part of the results):

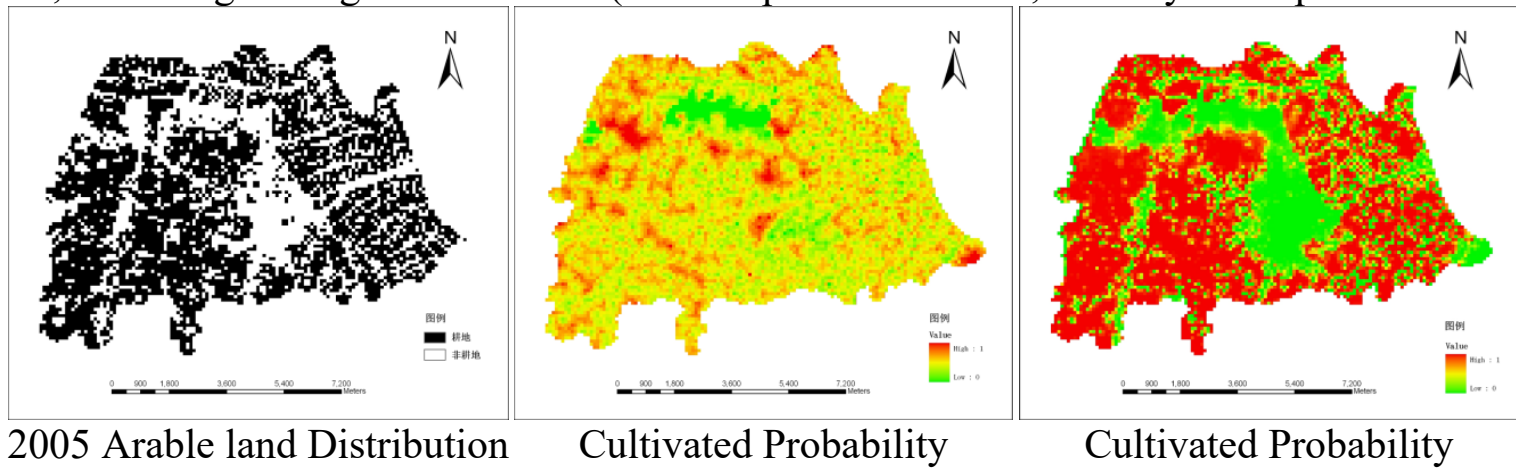

Pattern of Menghe Town Distribution Based on OLS Distribution Based on GWR

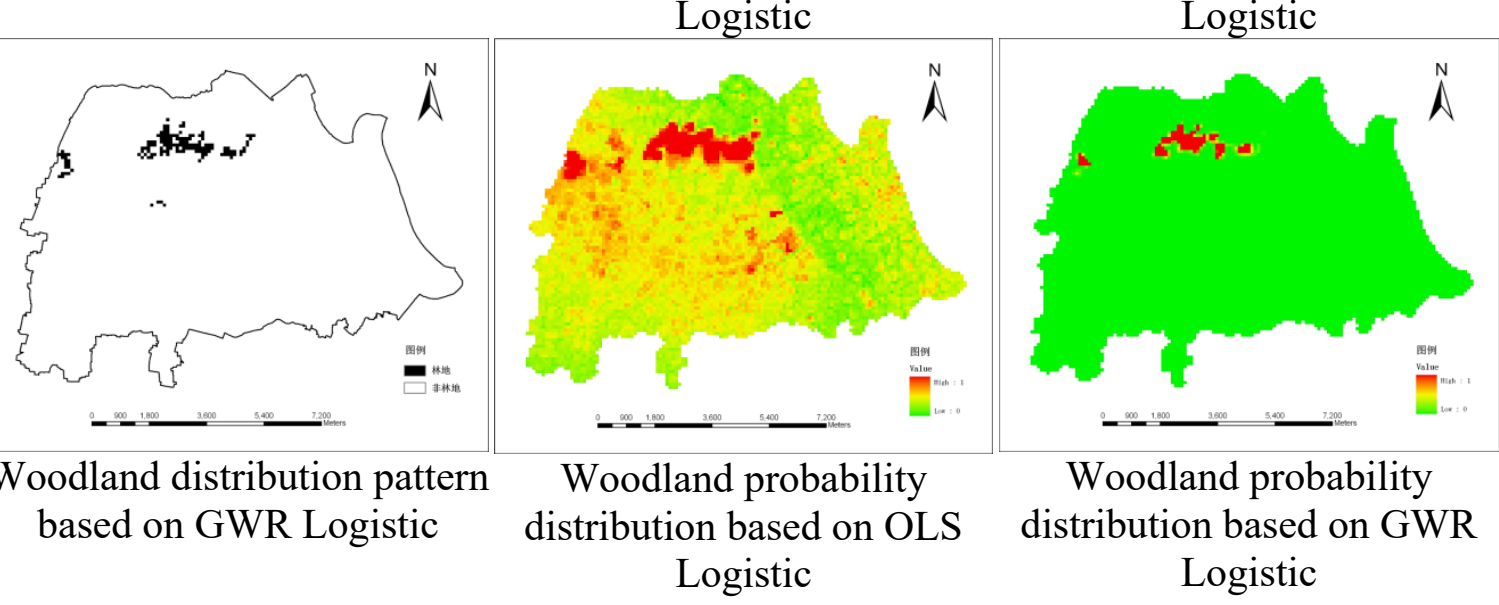

Figure 2: Patterns and Spatial Probability Simulation Results of Menghe Town of Changzhou City,

Farmland, Forest Land in 2005

According to Figure 2, comparing arable land with woodland simulate different probabilistic graphical model, we can find, with the real distribution of land use pattern compared to regression model based on GWR Logistic , the regression model relatively accurately reflected the true distribution of the corresponding land cover types. More importantly, GWR Logistic model based on a consideration of the driving factors of instability space, regression coefficient of the same driving factors vary in different grid cells, it is possible to reflect the various types of driving factors affect the degree of land use space differentiation characteristics, as shown in picture 4.

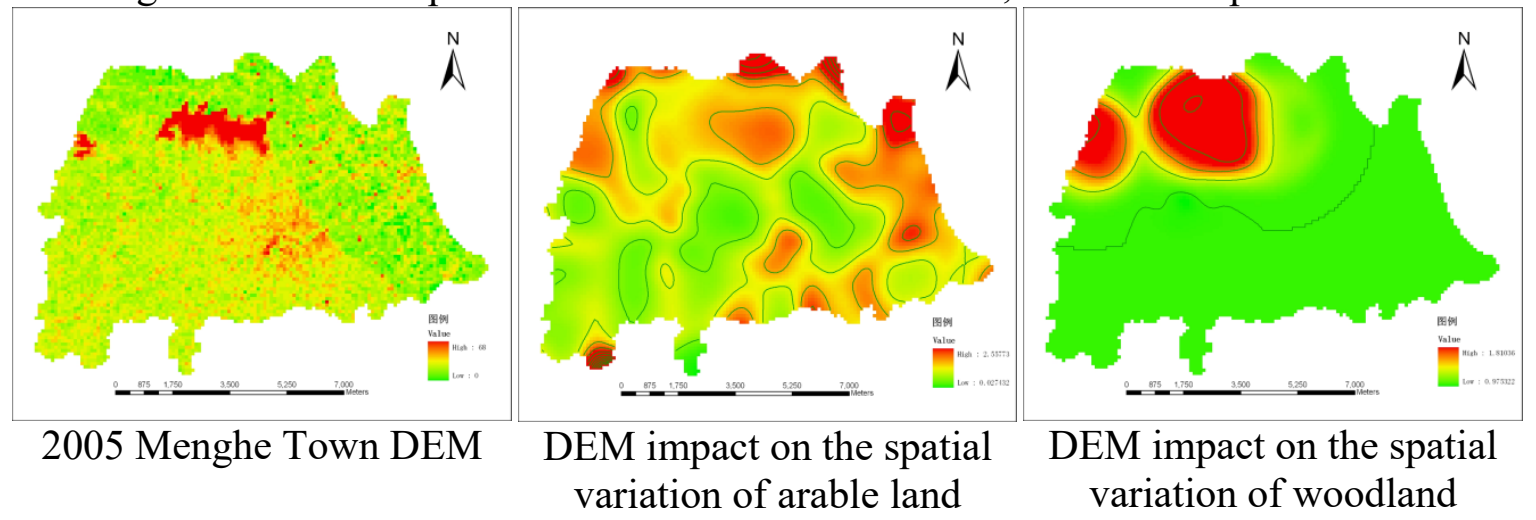

Figure 3 DEM driving factors of farmland, woodland affect the degree of spatial differentiation 
As can be seen from Figure 3, the high degree of influence Cheng Duigeng peak mostly distributed in north of town, east of town and the southwest corner of the town. Although town centre has elevation drop, but has little effect on the distribution of arable land, while the impact on the forest is concentrated in the northern and western mountains.

\section{Conclusions and Prospects}

\subsection{Conclusions}

Research started from the land use pattern and its driving factors in the quantitative relationship, analysing the land use pattern of spatial correlation, spatial instability and spatial heterogeneity, investigated land use pattern simulation modelling approach, established and implemented based on the geographical weighting the return of land use pattern simulation models. On this basis, as the study area the Menghe Town of Changzhou City in Jiangsu Province, studied the driving force of land use.

Studies suggested that, in the land use pattern and its driving factors in the regression model, whether they are land use types or driving forces, there are different degrees of spatial autocorrelation, and spatial structure of the distribution is not a single trend, exhibiting space instability and spatial heterogeneity. The GWR expresses itself the object correlation and heterogeneity parameter changes through additional space, reflecting the contribution of the sample regression equation in the space of differentiation. Therefore, it is necessary for GWR model to simulate land use pattern

Secondly, by employing land use pattern simulation model based on GWR it can analyse regional land use pattern and its drive space factor correlation, reflecting the natural and socioeconomic factors impact on land use patterns in different spatial locations, and have different constraints quantitatively under simulated land use pattern. The results provided a scientific basis for the analysis of the internal mechanism of land use change, rational development and use of land.

\subsection{Prospects}

At present, the land use pattern simulation study in China is still in its infancy, this study is the first to explore and try in geographically weighted regression model to simulate land use pattern of, due to limitations of time and research capabilities, the model for the resolution of only $100 \times 100 \mathrm{~m}$ were studied experimentally, the computational complexity of the model is relatively high. The future can be compared by testing different scales, to explore the land use pattern-dependent scale simulation model based on geographic weighted regression and to find the most appropriate scale.

\section{Acknowledgements}

This work was financially supported by Projects Funded by Top-notch Academic Programs Project of Jiangsu Higher Education Institutions (PPZY2015C203), Innovation Project of JiangSu Province, the Natural Science Foundation of Jiangsu Province No. BK20141033, the Open Project of Key Laboratory of Police Geographic Information Technology, Ministry of Public Security No. 2016LPGIT04, National Students' innovation and entrepreneurship training program No. 201610329003z, and Students' innovation and entrepreneurship training program of Jiangsu Police Institute No. 43y6201614. 


\section{References}

[1]Chenyou Qi, Yang Peng. Progress in the international land cover change research [J] land. Economic Geography, 2001, 21(1): 95-100

[2]Verburg P.H. Modeling the Spatial Dymatics of Regional Land Use: The CLUE-S Model [J]. Environment Management, 2002, 30(3):391-405.

[3]Verburg P.H., SoepboerW, Veldkamp A, et al.. Land use change modeling at the regional scale: The CLUE-S Model [J]. Environmental Management, 2002, 30: 391- 405.

[4]Gobin A, Campling P, Feyen J. Logistic modeling to derive agricultural land use determinants: a case study from south-eastern Nigeria [J]. Ecosystems and Environment, 2002, 89:213-228.

[5]Pendulum Wanqi, Yan Jianzhong, Zhang Yili Dadu River upstream of the Land Use / Land Cover Change and Driving Forces [J] Advances in Geographic Sciences, 2004, 23 (1): 71-78.

[6]Bakkera M.M,Goversb G, Kosmasc C, Vanackera V, van Oostb K, Rounsevella M. Soil erosion as a driver of land-use change. Agriculture, Ecosystems and Environment,2005,105:467-481.

[7]Zhang anding, Li deyi northern coast of Shandong Peninsula Land Use Change and Driving Force - A Case Study of Longkou City [J] Economic Geography 2007, 27 (6): 7001-0101.

[8]Shabana forest, Bo based farming-pastoral zone Logistic Regression Model Driving Force Analysis [J] Geography Land Use, 2008, 27 (2): 294-304.

[9]Fotheringham A. S., Chris Brunsdon, Martin Charlton Geographically weighted regression [M] U.K. West Sussex:. John Wiley \& Sons Ltd, 2002.

[10]Pontius R Gil, Schneider Laura C. Land-cover change model validation by an ROC method for the Ipswich watershed, Massachusetts, USA Agriculture, Ecosystems and Environment, 2001, 85: 239-248

[11]GWR model of urban residential land price spatial structure research [D] Luo Gang Hui. Based on Zhejiang University PhD thesis, 2007. 